\section{Níveis de chumbo em colostro humano: um estudo no Vale do Paraíba}

\section{Lead content in human colostrum: a study in the Paraíba Valley}

Luiz Fernando C. Nascimento 1 Hélcio José Izário Filho 2 Eduardo de Oliveira Baltazar 3

1 Disciplina de Informática Médica. Faculdade de Medicina. Universidade de São Paulo. Av. Dr. Arnaldo, 455. São Paulo, SP, Brasil. CEP. 01.246-904.E-mail: lfc@unitau.br

2,3 Faculdade de Engenharia Química de Lorena. Lorena, SP, Brasil

\begin{abstract}
Objectives: to estimate lead content in human colostrum in the Vale do Paraíba.

Methods: this is a cross sectional study performed in Taubaté, São Paulo, in the months of October and November of 2003, with mothers who delivered at the University Hospital of Taubaté. Colostrum was collected in an adequate tube and chilled to -200 C. Independent variables were mothers' age, parity, newborn age and pregnancy term. Lead was quantified by use of the atomic absorption spectrometry technique, in graphite furnace. For statistical analysis the Student " $t$ " test was used and in case of data not being normally distributed, the $U$ test of Mann-Whitney was applied, for continuous variables Pearson correlation was used for statistical analysis. Significance level adopted was alpha $=5 \%$.

Results: lead was detected in 72 samples of the 76 studies (94.7\% of the samples with IC95\%: 88,2\%98,4\%); median value found was $154,4 \mu \mathrm{g} / \mathrm{L}$ $(d p=173,7 \mu g / L)$. No correlation or differences were determined between lead content and the variables studied.

Conclusions: the values found are higher than the ones described in the literature and necessary to identify the possible sources of this heavy metal in the environment.
\end{abstract}

Key words Lead, Colostrum, Atomic absorption, Spectrometry, Milk, human

\section{Resumo}

Objetivos: estimar os valores de chumbo em colostro humano, no Vale do Paraíba.

Métodos: trata-se de um estudo transversal, realizado em Taubaté, São Paulo, nos meses de outubro e novembro de 2003, com mães que deram à luz no Hospital Universitário de Taubaté. O colostro foi coletado em tubo apropriado e resfriado a -200 C. As variáveis independentes foram idade materna, paridade e peso do recém-nascido e idade gestacional. O chumbo foi quantificado utilizando-se a técnica de espectrometria de absorção atômica, por forno de grafite. Para análise estatística utilizou-se o teste " $t$ " de Student $e$ quando os dados não tinham distribuição normal, o teste $U$ de Mann-Whitney; para as variáveis contínuas foi utilizada a correlação de Pearson. $O$ nível de significância adotado foi alfa $=5 \%$.

Resultados: o chumbo foi detectado em 72 amostras das 76 estudadas $(94,7 \%$ das amostras com IC $95 \%$ : 88,2\%-98,4\%); o valor médio encontrado foi $154,4 \mu \mathrm{g} / \mathrm{L}$ $(d p=173,7 \mu \mathrm{g} / \mathrm{L})$. Não houve correlação nem diferenças entre os valores de chumbo e as variáveis estudadas.

Conclusões: os valores encontrados estão acima dos descritos na literatura e torna-se necessária a identificação de possíveis fontes desse metal pesado no meio ambiente.

Palavras-chave Chumbo, Colostro, Absorção atômica, Espectrometria, Leite humano 


\section{Introdução}

Indiscutivelmente o leite materno é a melhor forma de se alimentar o recém-nascido, recomendado como maneira exclusiva de alimentação até os seis meses de vida. Porém, além de nutrientes e minerais essenciais necessários para o adequado crescimento da criança, o leite materno pode transportar metais pesados, como chumbo, que apresenta papel de toxicidade.

O chumbo é encontrado como poluente ambiental pela emissão industrial, principalmente por fábricas de baterias, incineradores e, também, por ingestão de alimentos contaminados. Mesmo em baixas concentrações pode comprometer o sistema nervoso, sangue e rins. Gundaker et al.,1 na Áustria, encontraram níveis de chumbo no leite materno de $1,63 \mu \mathrm{g} / \mathrm{L}$, estando esses níveis associados com o relato de fumo, consumo de peixe e o fato da mães residirem em cidade industrial.

Um estudo realizado no México ${ }^{2}$ mostrou valores médios de chumbo no leite humano de $62 \mu \mathrm{g} / \mathrm{L}$, sendo que em estudo colaborativo em seis países, a média de chumbo no leite humano variou entre 2,0 e $17,8 \mu \mathrm{g} / \mathrm{L} .{ }^{3}$ Hallén et al. 4 encontraram valores entre $0,1 \mu \mathrm{g} / \mathrm{L}$ e $2,2 \mu \mathrm{g} / \mathrm{L}$ de chumbo com valor médio de $0,7 \mu \mathrm{g} / \mathrm{L}$, sendo que os valores eram diferentes caso as mães vivessem próximas a fundições.

Durante os últimos anos fontes de contaminação ambiental por chumbo têm diminuído tanto pela abolição de chumbo na gasolina como também pelo fato de serem banidas as soldas em embalagens de alimentos e bebidas. A exposição dietética das crianças caiu de $10 \mu \mathrm{g} / \mathrm{kg}$.d, em 1980 para menos de $1 \mu \mathrm{g} / \mathrm{kg} . \mathrm{d} .^{5}$

A quantidade de chumbo no leite materno parece não sofrer grandes alterações e não segue um padrão específico em intervalos de até um ano de seguimento. 6,7 Essas quantidades parecem cair mais rapidamente caso a mãe resida em zona rural e, ao contrário, havia uma tendência de elevação desses valores se a mãe residisse em zona urbana. ${ }^{8}$

Além da contribuição materna durante a gravidez através da migração do chumbo dos ossos para o sangue e com conseqüente passagem placentária, o recém-nascido pode ingerir chumbo através do leite materno. Em seres humanos, Gulson et al. ${ }^{9}$ mostraram que havia uma mobilização aumentada e sustentada do chumbo dos ossos durante a lactação, quando comparada com a gravidez. Sowers et al. 10 encontraram valores médios de $6,1 \mu \mathrm{g} / \mathrm{L}$, o qual atribuíram, pelo menos em parte, a uma mobilização de chumbo dos ossos, no entanto sem que esses valores se constituíssem em problemas de saúde para o recém-nascido.

$\mathrm{O}$ interesse pelos valores de chumbo em leite no Brasil surgiu pela contaminação ambiental no Vale do Paraíba paulista por uma indústria de lingotes de chumbo, ${ }^{11}$ mas inexistem, no Brasil, trabalhos quantificando chumbo no colostro.

Assim, o objetivo deste trabalho é estimar os valores de chumbo no colostro humano, na região Sudeste do Brasil.

\section{Métodos}

Trata-se de um estudo transversal, envolvendo mães que deram à luz no Hospital Universitário de Taubaté, São Paulo, Brasil, nos meses de outubro e novembro de 2003. Taubaté situa-se entre os estados de São Paulo e Rio de Janeiro, tem um importante parque industrial e uma população estimada em 250.000 habitantes.

Os critérios de inclusão eram que a gravidez se tivesse desenvolvido sem intercorrências, o trabalho de parto tivesse transcorrido sem anormalidades, o recém-nascido não pertencesse a grupos de risco nem tivesse que ser internado em Unidade de Terapia Intensiva Neonatal e que houvesse facilidade de obtenção do colostro. O grupo de mães incluídas fizeram o pré-natal no Hospital Universitário e eram usuárias do Sistema Único de Saúde.

As amostras, contendo $5 \mathrm{~mL}$ de colostro, foram colhidas ainda no hospital, nas primeiras 24 horas pós-parto, através de expressão manual, após limpeza do mamilo com água deionizada. Os tubos de polietileno, previamente tratados com ácido nítrico a $10 \% \mathrm{v} / \mathrm{v}$, foram armazenados a $-20^{\circ} \mathrm{C}$.

A espectrometria de absorção atômica com forno de grafite foi usada para determinar o elemento metálico de interesse, utilizando-se o espectrômetro marca Perkin Elmer, modelo Aanalyst 800, equipado com corretor, utilizando o efeito Zeeman.

As amostras, em duplicata, foram digeridas em cápsulas de Teflon, utilizando uma mistura ácida de $\mathrm{HCl}$ e $\mathrm{HNO}_{3}$. As cápsulas de Teflon foram introduzidas em cápsulas de alumínio, resistente a pressão e temperatura, onde eram aquecidas em forno resistivo de aço inox com controle de temperatura. Os valores obtidos para cada amostra representaram a média dessas duas amostras.

Para o estudo da exatidão do método foi usado um material de referência certificado de leite, distribuído por National Institute of Standards and Technology, RM 8435, tendo uma alta recuperação (>95\%) com um desvio padrão relativo médio de 2,0. 
Para este estudo, caso os valores encontrados para o chumbo estivessem abaixo do limite de quantificação (LOQ), eram considerados zero.

As variáveis independentes foram: idade materna, categorizada em adolescente, até 19 anos de idade e adulta as demais; tabagismo materno; paridade, categorizada em 0 e 1 filho e 2 ou mais filhos; peso do recém-nascido obtido de forma contínua e depois categorizado em baixo peso e peso normal; idade gestacional obtida em dias, pela técnica de Capurro, e depois categorizada em prétermo, quando inferior a 259 dias e de termo. Essas informações foram obtidas diretamente da mãe ou da ficha obstétrica, imediatamente após o parto.

Hábitos alimentares foram obtidos através de um questionário aplicado às mães, relativo aos últimos dois meses de gestação, onde constava relato sobre a ingestão de arroz, feijão, carnes de vaca, frango ou peixe, leite, pão, verduras e legumes comercializados na região. A informação sobre profissão materna foi obtida diretamente da mãe.

Foram feitas correlações entre as variáveis quantitativas e os resultados encontrados para chumbo, utilizando-se a correlação de Pearson. As médias foram comparadas utilizando-se a técnica $t$ de Student, e nos casos em que os dados não apresentassem distribuição normal foi utilizado o teste nãoparamétrico de Mann-Whitney. Os valores encontrados para o chumbo foram apresentados sob forma de tabela e de gráfico box-and-whisker plot e agrupados em até $50 \mu \mathrm{g} / \mathrm{L}$, de 50 a $100 \mu \mathrm{g} / \mathrm{L}$, de 100 a $200 \mu \mathrm{g} / \mathrm{L}$, de 200 a $300 \mu \mathrm{g} / \mathrm{L}$ e acima de $300 \mu \mathrm{g} / \mathrm{L}$. O nível de significância adotado neste estudo foi alfa $=0,05$.

Todas as mães incluídas no presente estudo eram moradoras da área urbana de Taubaté, e, após exposição dos motivos da pesquisa, assinaram o termo de consentimento livre e esclarecido. A amostra foi do tipo de conveniência.

O estudo foi aprovado pelo Comitê de Ética do Hospital Universitário da Universidade de Taubaté.

\section{Resultados}

Foram estudadas 76 amostras de colostro humano. A idade materna média foi 24,8 anos $(\mathrm{dp}=6,8)$ variando entre 15 e 42 anos. Havia história positiva de tabagismo em seis mães e negativa em 70 .

A paridade média encontrada nessa amostra foi 1,6 filhos $(\mathrm{dp}=1,5)$, variando entre 0 e 7 filhos; o peso médio dos $\mathrm{RN}$ foi $3.000 \mathrm{~g}(\mathrm{dp}=452,4)$, com valores mínimo e máximo de $2.165-4.250 \mathrm{~g}$ respectivamente e a idade gestacional média foi 269,2 dias (dp=10,1), com valores mínimo e máximo de 231 294 dias.

Todas as mães exerciam atividades domésticas. Os hábitos alimentares das mães consistiam na ingestão regular de arroz, feijão, carnes de vaca ou de frango, legumes e verduras cultivados e comer-

Figura 1

Box-and whisker plot do conteúdo de chumbo $(\mu \mathrm{g} / \mathrm{L})$ em 72 amostras de colostro de mães.Taubaté, São Paulo.

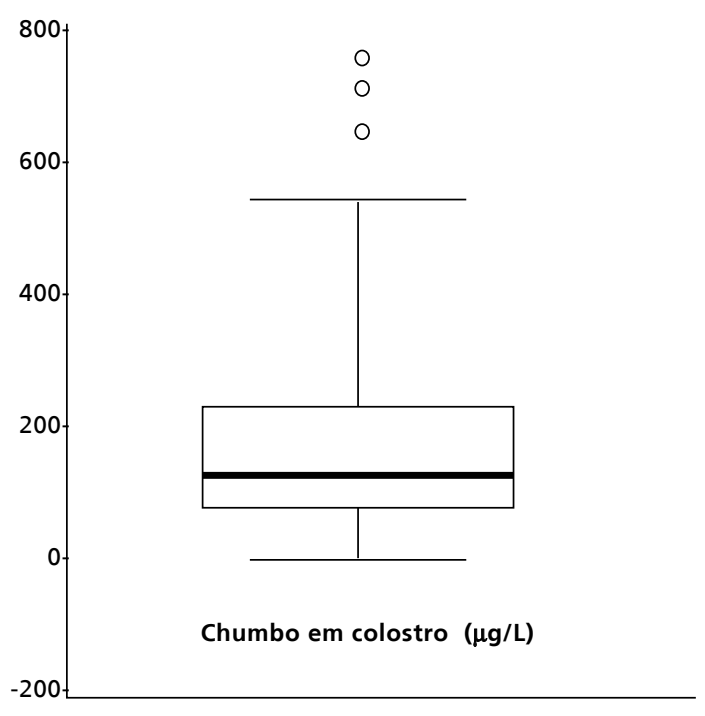

Figura 2

Distribuição das amostras de colostro humano segundo conteúdo de chumbo, em $\mu \mathrm{g} / \mathrm{L}$, categorizado em classes.

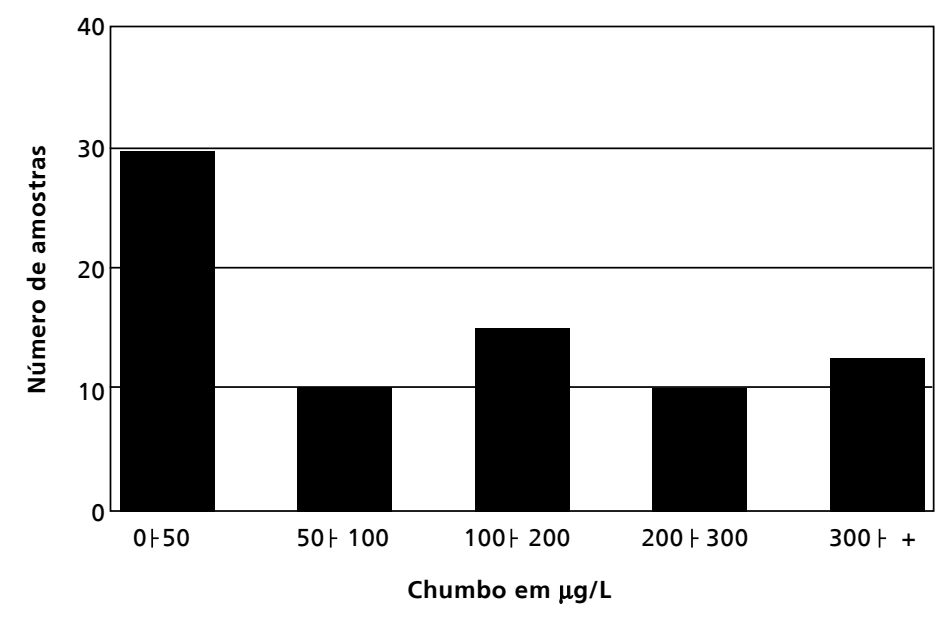


Tabela 1

Valores médios ( $+\mathrm{dp}$ ), do chumbo, em $\mu \mathrm{g} / \mathrm{L}$, encontrados no colostro de 76 mães, segundo idade materna, peso ao nascimento, idade gestacional e paridade. Taubaté, São Paulo.

\begin{tabular}{|c|c|c|c|c|}
\hline & Média & \pm & $d p$ & $p$ \\
\hline \multicolumn{5}{|l|}{ Idade materna } \\
\hline$<20$ anos $(23)$ & 286,0 & \pm & 409,0 & $0,18 *$ \\
\hline$>=20$ anos (53) & 196,0 & \pm & 316,0 & \\
\hline Baixo peso (12) & 97,3 & \pm & 89,7 & $0,37 * *$ \\
\hline Peso normal (64) & 165,3 & \pm & 179,9 & \\
\hline Pré-termo (9) & 151,3 & \pm & 118,0 & $0,89 * *$ \\
\hline Termo (67) & 159,9 & \pm & 177,3 & \\
\hline 0 e 1 filho (41) & 157,0 & \pm & 262,0 & 0,31 ** \\
\hline 2 ou mais filhos (35) & 171,0 & \pm & 159,0 & \\
\hline
\end{tabular}

*Teste " $\mathrm{t}$ " de Student; **Teste Mann-Whitney

Tabela 2

Coeficientes de correlação de Pearson entre os valores de chumbo quantificado no colostro de 76 puérperas, em $\mu \mathrm{g} / \mathrm{L}$, e as variáveis peso ao nascimento, idade materna e idade gestacional. Taubaté, São Paulo.

\begin{tabular}{lc}
\hline Média (dp) & $\begin{array}{c}\text { Coeficiente } \\
\text { de correlação }\end{array}$ \\
\hline Peso ao nascer & $-0,06^{*}$ \\
Idade materna & $-0,11^{*}$ \\
Idade gestacional & $-0,15^{*}$ \\
Paridade & $0,05^{*}$ \\
\hline
\end{tabular}

$* p>0,05$

Tabela 3

Valores médios de chumbo, encontrados neste e em outros estudos, segundo o tipo de leite analisado.

\begin{tabular}{lc}
\hline & $\mathrm{Pb}(\mu \mathrm{g} / \mathrm{L})$ \\
\hline Gundacker et al.1 [colostro] & 1,63 \\
Nahimira et al.2 [leite maduro] & 62,4 \\
Hallén et al.4 [leite maduro] & 0,9 \\
Turan et al.12 [colostro] & 14,6 \\
Krachler et al.13 [colostro] & 3,5 \\
Tripathi et al.14 [leite maduro] & 1,9 \\
Ettinger et al.15 [leite maduro] & 1,1 \\
Este estudo [colostro] & 154,4 \\
\hline
\end{tabular}

cializados normalmente na região, e leite pasteurizado; não houve relato de consumo de vinho ou destilados e também de consumo de peixe, rotineiramente.

O chumbo, $(\mathrm{LOQ}=0,41 \mu \mathrm{g} / \mathrm{L})$, foi detectado em 72 amostras de colostro, representando $94,7 \%$ das amostras (IC95\%: 88,2\%-98,4\%). Quando detectado, variou entre $1,0 \mu \mathrm{g} / 1$ e $742,0 \mu \mathrm{g} / \mathrm{L}$. Os valores médios encontrados para o chumbo, considerando zero se os valores fossem inferior ao limite de quantificação, foi $154,4 \mu \mathrm{g} / \mathrm{L}(\mathrm{dp}=173,7)$. A mediana dessa distribuição foi $92,0 \mu \mathrm{g} / \mathrm{L}$, sendo que o primeiro quartil apresentou valor de $26,0 \mu \mathrm{g} / \mathrm{L}$ e o terceiro quartil, $225,5 \mu \mathrm{g} / \mathrm{L}$ e três valores aberrantes (Figura 1).

Foram encontradas 29 amostras com valores até $50 \mu \mathrm{g} / 1,10$ amostras contendo chumbo na quantidade entre 50 a $100 \mu \mathrm{g} / \mathrm{L}, 15$ amostras com valores entre 100 a $200 \mu \mathrm{g} / \mathrm{L}, 10$ entre 200 a $300 \mu \mathrm{g} / \mathrm{L}$ e 12 amostras contendo chumbo acima de $300 \mu \mathrm{g} / \mathrm{L}$ (Figura 2).

A Tabela 1 mostra os valores médios de chumbo e as variáveis. Pode ser observada a inexistência de diferenças, com significância estatística, entre os conteúdos médios de chumbo e as variáveis já categorizadas: peso ao nascer, tempo de gestação, paridade, idade materna.

O coeficiente de correlação entre os valores de chumbo e idade materna foi $r=-0,11$; entre os valores de chumbo e peso do $\mathrm{RN}, \mathrm{r}=-0,06$; os valores do coeficiente de correlação entre os valores de chumbo e idade gestacional e paridade foram $r=-0,15$ e 0,05 , respectivamente, sem significância estatística (Tabela 2).

Não houve associação com significância estatística entre as classes de valores do chumbo e as categorias baixo peso e peso normal e também com a idade gestacional categorizada em termo e prétermo.

A Tabela 3 mostra os valores de chumbo encontrados neste e em outros estudos.

\section{Discussão}

Trata-se do primeiro estudo no Brasil sobre o conteúdo de chumbo no colostro humano. Também, na literatura mundial, são poucos os trabalhos sobre esse tema. O tamanho da amostra, 76 mães, situouse acima do tamanho amostral de vários estudos. $1,4,10,12-14$

O que mais chamou a atenção foram os valores obtidos nesta pesquisa. A coleta do colostro foi realizada de maneira tal que se afastasse o risco de conta- 
minação externa por metais, sendo sempre realizada por único profissional médico que havia sido treinado, e em tubos previamente tratados.

Mesmos com os cuidados tomados, os achados estão muito acima dos encontrados por outros autores, sendo necessária a identificação de possíveis fontes de contaminação. 1,2,4,12-15

Não foram encontradas correlações entre os níveis de chumbo no colostro e idade materna. Apesar de se esperar que mães mais velhas apresentassem valores maiores, a correlação encontrada foi negativa; quando categorizada em dois grupos, o grupo mais jovem apresentou níveis mais elevados de chumbo; ambas as análises não apresentavam significância estatística. Esse fato também foi encontrado por Ettinger et al. 15

Quanto à paridade, as mães com 0 ou 1 filho apresentaram níveis maiores de chumbo, ao contrário dos achados de Ettinger et al. 15 Para esse fato não foi possível encontrar uma explicação razoável, pois seria de se esperar que uma quantidade aumentada fosse detectada em multíparas, uma vez que já poderia ter ocorrido a mobilização do chumbo do osso para o sangue. Mesmo após ser estratificada pela idade materna, não foram encontradas diferenças.

Tanto para o peso ao nascer como para a idade gestacional, este estudo não mostrou correlação ou associação com os níveis de chumbo, mesmo com o conteúdo de chumbo no colostro agrupado em classes. Não foram encontrados, na literatura consultada, dados sobre uma possível associação ou correlação entre chumbo e essas duas variáveis.

Considerando uma ingestão de colostro, por um recém-nascido, de $600 \mathrm{~mL} / \mathrm{d}$, pode-se estimar uma ingestão média diária ao redor de $90 \mu \mathrm{g}$ de chumbo. Para um recém-nascido com peso de $3 \mathrm{~kg}$, a ingestão seria de $30 \mu \mathrm{g} / \mathrm{kg}$.d. Esses valores estão acima do permitido $^{3}$, que é $3,6 \mu \mathrm{g} / \mathrm{d}$.

A presença de chumbo no colostro poderia ser decorrente da poluição atmosférica ou de água, por indústrias, pois fontes conhecidas como vinho e peixe não fazem parte do cardápio rotineiro dos habitantes desta região. No entanto, não foi possível identificar as fontes de contaminação.

Esses valores elevados podem traduzir os níveis de chumbo no sangue materno e também no sangue do recém-nascido. Ettinger et al. 16 encontraram uma forte correlação entre os níveis de chumbo no leite de nutrizes um mês após o parto e os níveis de chumbo no sangue dessas mães e também dos recém-nascidos. No referido estudo, realizado na Cidade do México, os autores mostraram que para cada $2 \mu \mathrm{g} / \mathrm{L}$ de chumbo a mais no leite materno, havia um aumento de $0,82 \mu \mathrm{g} / \mathrm{dL}$ de chumbo no sangue do lactente.

Outra explicação para valores elevados de chumbo no colostro, considerando que estas puérperas não eram trabalhadoras da indústria, seria a mobilização do chumbo ósseo para o sangue materno da nutriz. Tal fato é corroborado por Abadin et al.17 que encontraram níveis detectáveis de chumbo em mães sem qualquer exposição ambiental. É sabido que na lactação ocorre uma substancial redistribuição de cálcio através da mobilização do cálcio ósseo. Estima-se que 5\% da massa óssea é mobilizada durante a lactação; dessa forma, o chumbo acumulado nos ossos dessa puérpera, até a suspensão do uso de chumbo na gasolina e da abolição de alimentos envasados com utilização de soldas, pode ser liberado para o sangue e excretado pelo leite materno.9,18 Essa transferência é ativa do sangue para o leite e foi estimada em até $300 \%$ maiores no leite materno que no sangue materno. 10 Neste houve uma correlação positiva entre a paridade e os valores de chumbo, porém sem significância estatística.

Assim, o leite materno e, em especial o colostro, pode ser um estimador da plumbemia materna e também do lactente durante o aleitamento materno, visto que a coleta é através de um método não-invasivo, sem risco para a mãe. A limitação para esse monitoramento é a técnica de análise química, envolvendo tecnologia que não é encontrada rotineiramente, exceto em institutos de pesquisa, além do cuidado para se evitar a contaminação do colostro por chumbo. Deve ser ressaltado, também, que não há estudos estimando a plumbemia em mães nesta região.

Outro ponto importante a ser considerado é a exposição do recém-nascido a alimentos, no caso o leite materno, que podem apresentar considerável quantidade de chumbo. No entanto, os benefícios do aleitamento materno devem ser julgados por um conjunto mais complexo de dados além da biodisponibilidade do chumbo, a não ser que a exposição materna seja muito grande, tais como morar próximo a fundições, uso de tintas com freqüência, uso de material cerâmico para a alimentação, cosméticos e fontes alimentares.

Assim, este primeiro trabalho realizado no Brasil, ainda que regionalizado, mostra a presença de chumbo no colostro humano e evedencia que estes valores estão muito acima dos encontrados em outros estudos e, além disto, abre perspectivas para identificação de possíveis fontes ambientais contendo esse metal que, em contato com as mães, são responsáveis por altos teores no leite materno. 


\section{Referências}

1. Gundacker C, Pietschnig B, Wittmann KJ, Lischka A, Salzer H, Hohenauer L, Schuster E. Lead and mercury in breast milk. Pediatrics 2002; 110: 873-8.

2. Nahimira D, Saldivar L, Pustilnik N, Carreon GJ, Salina ME. Lead in human blood and milk from nursing women living near a smelter in Mexico city. J Toxicol Environ Health 1993; 38: 225-32.

3. WHO (World Health Organization) IAEA (International Atomic Energy Agency) Minor and trace elements in breast milk, Collaborative Study. Geneve; 1989.

4. Hallén IP, Jorhem L, Lagerkvist BJ, Oskarsson A. Lead and cadmium levels in human milk and blood. Sci Total Environ 1995; 166: 149-55.

5. Gulson BL, Jameson CW, Mahaffey KR, Mizon KJ, Patison P, Law AJ, Korsch MJ, Salter MA. Relationships of lead in breast milk to lead in blood, urine, and diet of the infant and mother. Environ Health Perspect 1998; 106: 667-74

6. Ong CN, Phoon WO, Law HY, Tye CY, Lim HH. Concentrations of lead in maternal blood, cord blood, and breast milk. Arch Dis Child 1985; 60:756-9.

7. Huat LH, Zakariya D, Hoon K. Lead concentrations in breast milk of Malaysian urban and rural mothers. Arch Environ Health 1983; 38: 205-9.

8. Sternowsky HJ, Wessolowski R. Lead and cadmium in breast milk. Arch Toxicol 1985; 57: 41-5.

9. Gulson BL, Mahaffey KR, Jameson CW, Mizon KJ, Korsch MJ, Cameron MA, Eisman JA. Mobilization of lead from the skeleton during the postnatal period is larger than during pregnancy. J Lab Clin Med 1998;131: 324-9 .

10. Sowers MFR, Scholl TO, Hall G, Jannausch ML, Kemp $\mathrm{FW}, \mathrm{Li} \mathrm{X}$ et al. Lead in breast milk and maternal turnover. Am J Obstet Gynecol 2002; 187: 770-6.
11. Okada IA, Sakuma AM, Maio FD, Dovidauskas S, Zenebon O. Avaliação dos níveis de chumbo e cádmio em leite em decorrência de contaminação ambiental na região do Vale do Paraíba, Sudeste do Brasil. Rev Saúde Pública 1997; 31 : 140-3.

12. Turan S, Saygi S, Kiliç Z, Acar O. Determination of heavy metal content in human colostrum samples by electrothermal atomic absorption spectrophotometry. J Trop Pediatr 2001; 47: 81-5.

13. Krachler M, Rossipal E, Micetic-Turk D. Trace element transfer from the mother to the newborn - investigations on triplets of colostrum, maternal and umbilical cord sera. Eur J Clin Nutr 1999; 53: 486-94.

14. Tripathi RM, Raghunath R, Sastry VN, Krishnamoorthy TM. Daily intake of heavy metals by infants through milk and milk products. Sci Total Environ 1999; 227: 229-35.

15. Ettinger AS, Téllez-Rojo MM, Amarasiriwardena C, Gonzalez-Cossio T, Peterson KE, Aro H, Hu H, HernandezAvila M. Levels of lead in breast milk and their relation to maternal blood and bone lead levels at one month postpartum.. Environ Health Perspect 2004; 112: 926-31.

16. Ettinger AS, Téllez-Rojo MM, Amarasiriwardena C, Bellinger D, Peterson KE, Schwartz J, Hu H, HernandezAvila M. Effect of breast milk lead on infant blood levels ao one month of age. Environ Health Perspect 2004; 112: 1381-5.

17. Abadin HG, Hibbs BF, Pohl HR. Breast-feeding exposure of infants to cadmium, lead, and mercury: a public health viewpoint. Toxicol Ind Health 1997; 13: 495-517.

18. Sowers MFR. Pregnancy and lactation as risk factors for subsequent bone loss and osteoporosis. J Bone Miner Res 1996; 11: 1052-60.

Recebido em 4 de julho de 2005

Versão final em 27 de janeiro de 2006

Aprovado em 6 de fevereiro de 2006 